\title{
Synthetic rutile derived titanium alloy development utilising the Metalysis Process and field assisted sintering technology
}

\author{
Simon J. Graham ${ }^{1}$, Lyndsey L. Benson ${ }^{2}$, Martin Jackson ${ }^{1}$ \\ ${ }^{1}$ University of Sheffield, Department of Materials Science and Engineering, Sir Robert Hadfield Building, Mappin Street, Sheffield, S1 \\ 3JD, UK; ${ }^{2}$ Metalysis Ltd, Unit 4 R-evolution@the AMP, Brindley Way, Catcliffe, Rotherham, S60 5FS, UK
}

Email: sjgraham1@sheffield.ac.uk

\begin{abstract}
$\underline{\text { Abstract }}$
Utilising novel extraction and processing technologies allows for the realisation of an alternative titanium alloy production route, with many benefits over the traditional Kroll-based one. The route proposed has the potential to reduce the cost of titanium and offers the ability to create alloys which are difficult to make conventionally. It combines the Metalysis Process, an electrolytic metal extraction technique, with field assisted sintering technology (FAST), a rapid and effective solid-state sintering technique. The Metalysis Process reduces metal oxide powders directly into metal powders, which can then be consolidated using FAST. Using synthetic rutile (SR) as the feedstock, compared to pigment grade rutile and $\mathrm{TiCl}_{4}$, further reduces the cost of titanium produced via this route.
\end{abstract}

This research investigates the use of this route to create a range of pseudo-binary Ti-Fe alloys, by co-reducing SR with iron (III) oxide $\left(\mathrm{Fe}_{2} \mathrm{O}_{3}\right)$. Various techniques were used to analyse the feedstock, reduced alloy powders and consolidated material post-FAST to determine chemistry and microstructure.

\section{$\underline{\text { Introduction }}$}

The costs associated with current commercial titanium production prevent widespread use of the material, despite relative ore abundance. Titanium and its alloys have impressive properties and are desirable for a wide variety of applications, but inferior materials are often used preferentially due to their lower cost. Reducing prices significantly requires the realisation of an alternative production route which is becoming increasingly plausible with novel extraction and processing techniques. Combining the Metalysis Process with field assisted sintering technology (FAST) offers an alternative, entirely solid-state processing route (Figure 1). 


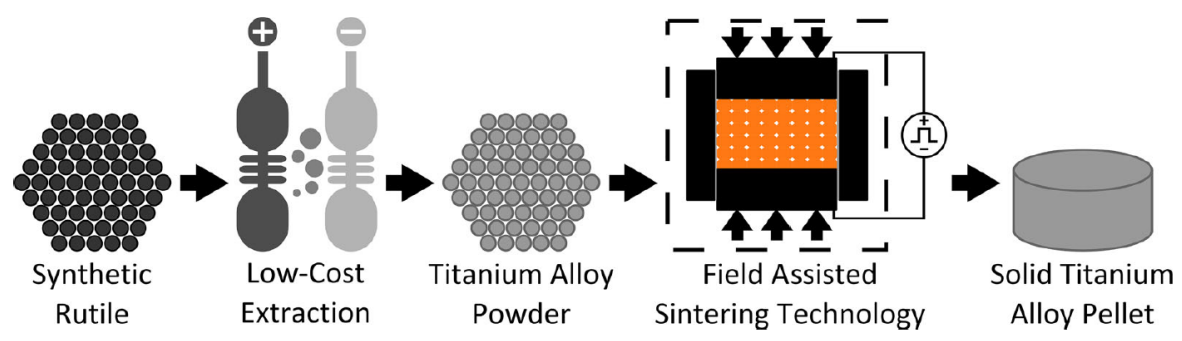

Figure 1: Proposed Ti extraction and processing route (small-scale)

The original incarnation of the Metalysis Process was known as the FFC process and was first reported in 2000 by researchers Fray, Farthing and Chen as an electrolytic method for extracting titanium metal from its oxide [1]. The electrolytic reduction involves using the metal oxide as the cathode of an electrochemical cell, along with a graphite anode in a molten salt electrolyte (usually $\left.\mathrm{CaCl}_{2}\right)$. An applied voltage causes ionisation of the oxygen at the cathode which diffuses into the electrolyte (Eq. 1). The oxygen ions then migrate through the electrolyte to combine with the carbon at the anode to produce $\mathrm{CO}$ and $\mathrm{CO}_{2}$ (Eq. $2 \& 3$ ). Therefore, overall the oxygen is transferred from the oxide to the carbon, leaving behind the base metal [2].

$$
\begin{gathered}
M_{x} O_{y}+2 y e^{-} \longrightarrow x M+y O^{2-} \\
C+2 O^{2-} \longrightarrow \mathrm{CO}_{2}+4 e^{-} \\
C+\mathrm{O}^{2-} \longrightarrow \mathrm{CO}+2 e^{-}
\end{gathered}
$$

The process has since been successfully done with many other metal and mixed-metal oxides, including tantalum, chromium, cerium, aluminium-scandium and for the production of high entropy alloys (HEAs) [3]-[7]. Most research has been on titanium however, due to the potential benefits this technology has over traditional Kroll extraction. Metalysis have since been working on optimising and scaling up the process to make the technology commercially viable. This includes the ability to directly reduce powder feedstock without need for compaction into preform pellets [2].

Utilising synthetic rutile (SR) can further reduce the cost of titanium production via this route. Ilmenite ore $\left(\mathrm{FeTiO}_{3}\right)$ is far more abundant and therefore cheaper than natural rutile $\left(\mathrm{TiO}_{2}\right)$, and is upgraded to $\mathrm{SR}\left(>95 \% \mathrm{TiO}_{2}\right)$ by an iron removal process [8]. Depending on the ilmenite source and exact processing conditions, impurity elements including Fe, Al and Mn are present in varying amounts. SR powder has been directly reduced using the Metalysis Process, and the reduction pathway fully characterised, producing a titanium alloy powder naturally containing these impurities as alloying elements [9].

Field assisted sintering technology (FAST), commonly known as spark plasma sintering, is an effective technique for consolidating a range of powder materials. It does this through a combination of uniaxial pressure and joule heating and facilitates rapid, solid-state 
sintering. It has been used with a range of titanium alloy powders and is generally seen as an intermediate step before forging and finish machining. This processing route has been developed under the term FAST-forge [10]. An SR derived powder direct from the Metalysis Process has already been consolidated using FAST to directly give an $\alpha+\beta$ microstructure and comparable mechanical properties to Ti-64 [11].

Given the Metalysis Process allows for the reduction of mixed-metal oxides, it is also possible to reduce SR alongside other oxides to produce alternative SR-derived alloys. Pseudo-binary Ti-Fe alloys could be made by reducing $\mathrm{SR}$ with $\mathrm{Fe}_{2} \mathrm{O}_{3}$, enhancing the Fe content which is already present in SR. Fe is the most effective $\beta$ phase stabilising element, however is not heavily used in titanium alloys due to segregation issues during vacuum melting. This can lead to $\beta$ rich regions, known as $\beta$ flecks and in extreme cases intermetallic TiFe formation due to its eutectoid behaviour [12]. Mo and V are often used as $\beta$ stabilisers instead as these issues do not occur due to them being isomorphous with Ti. They are expensive alloying additions however, further adding to the overall cost of many titanium alloys. The issues with $\mathrm{Fe}$ can be resolved using the proposed route. As both the reduction and consolidation occur in the solid-state and Fe is a fast diffuser in titanium, homogeneous alloys can be formed during consolidation [13].

Intermetallic TiFe formation does limit the amount of Fe that can be used in titanium alloys, however. Figure 2 shows that Fe has essentially no solid solubility in $\alpha$ and limited in $\beta$, as it is a $\beta$-eutectoid stabiliser [14]. TiFe formation is known to be slow however, despite being thermodynamically favourable and so can be avoided with a sufficient cooling rate [15]. Also, as Fe is a significantly more effective $\beta$ stabiliser than Mo and $\mathrm{V}$, a lower content is required to give the same stabilising effect.

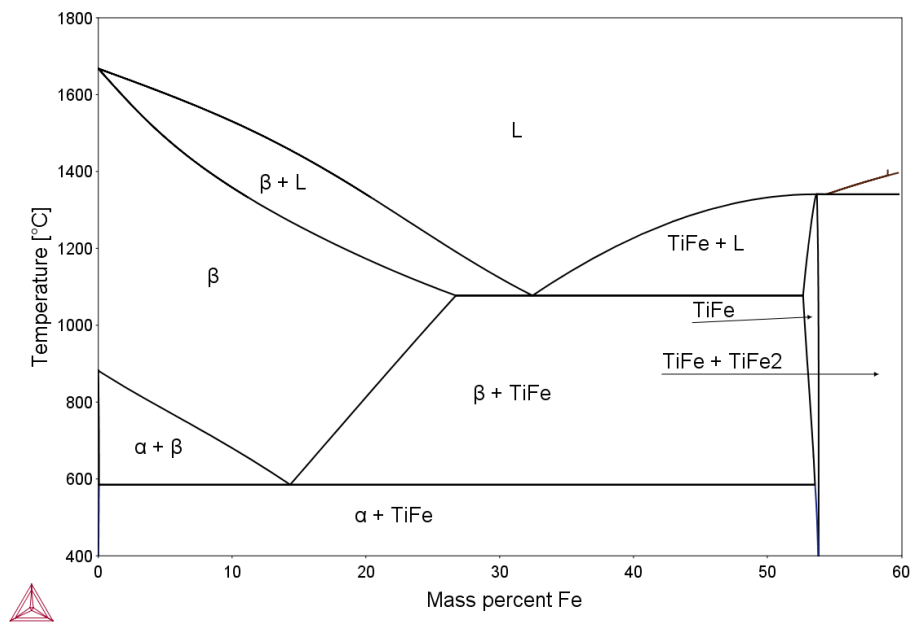

Figure 2: Ti-Fe phase diagram calculated using Thermocalc TTTI3 database [14]

Therefore, there is a possibility of making cheaper metastable $\beta$ alloys by using Fe as a major alloying element through this process. This has been investigated previously, with binary Ti-Fe alloys up to $7 \mathrm{wt} . \% \mathrm{Fe}$ having been produced using blended elemental powders and with no trace of intermetallic TiFe [16]-[18]. These were produced by mixing, cold pressing, then furnace sintering, however this leaves 
significant porosity. Powders from the Metalysis Process have the potential to be cheaper though as they are produced directly and FAST has shown to achieve consolidation to full density which is important for mechanical properties [19].

The aim of this work is therefore to make a range of alloys with differing Fe contents by co-reducing SR and $\mathrm{Fe}_{2} \mathrm{O}_{3}$. The powders are then consolidated using FAST and the bulk material properties analysed to investigate the effect of Fe content and compare to commercially available alloys.

\section{Experimental methods}

\section{Oxide preparation}

Following a treatment step, synthetic rutile powder (provided by Iluka Resources, $150-212 \mu \mathrm{m}$ ) was mixed with $\mathrm{Fe}_{2} \mathrm{O}_{3}$ powder (Sigma-Aldrich $<5 \mu \mathrm{m}$ ) using a Turbular mixer for 1 hour. 3 different oxide compositions were made in addition to standard SR containing $1,2.5$ and 5 wt. $\% \mathrm{Fe}_{2} \mathrm{O}_{3}$.

\section{Reduction and post-processing}

The 4 oxides were reduced simultaneously using a development cell at Metalysis ( $5 \mathrm{~kg}$ total oxide). The resulting material then underwent a cleaning and drying process to yield the alloy powders which were sieved to standard sizes of $75-500 \mu \mathrm{m}$.

\section{Consolidation}

The alloy powders were consolidated by FAST using an FCT Systeme GmbH type HP D25 spark plasma sintering furnace with a 20 mm graphite mould set. The parameters included a $100{ }^{\circ} \mathrm{C} / \mathrm{min}$ ramp rate to a maximum temperature of $1000{ }^{\circ} \mathrm{C}$, a dwell of 30 minutes and a force of $11 \mathrm{kN}(35 \mathrm{MPa}) .5 \mathrm{~g}$ of powder was used to create a disc of around $3 \mathrm{~mm}$ height. The discs were then cut perpendicular to the compression direction to expose a surface for metallographic preparation and analysis.

\section{Analyses}

The feedstock, alloy powders and consolidated material were all analysed using various techniques. Scanning electron microscopy (SEM) and was utilised for microstructural and morphology analysis. An FEI Inspect F50 and a JEOL JSM-6490LV were used with an acceleration voltage of $20 \mathrm{keV}$. Energy-dispersive X-ray spectroscopy (X-EDS) was also completed using an Oxford Instruments attachment. Powder chemistry was determined by inductively coupled plasma mass spectrometry (ICP-MS) using a Thermo X series 2 . Interstitial element contents were measured using an ELTRA ON-900 and an Eltra CS-800. 


\section{Results and discussion}

Characterisation of synthetic rutile powder feedstock prior to electrolytic reduction

Provided X-ray fluorescence (XRF) data of the SR showed that it contained $\sim 4$ wt. $\% \beta$ stabilising elements, mainly Fe, and $<1$ wt.\% Al. Backscattered SEM images of the treated SR powder particles alongside XRD analysis confirmed the presence of two phases, $\mathrm{TiO}_{2}$ (rutile) and $\mathrm{Fe}_{2} \mathrm{TiO}_{5}$ (pseudobrookite). Figure 3a shows a particle containing the two phases, with the $\mathrm{Fe}_{2} \mathrm{TiO}_{5}$ appearing brighter due to the heavier Fe. Some particles do not contain any $\mathrm{Fe}_{2} \mathrm{TiO}_{5}$ however and exhibit solely $\mathrm{TiO}_{2}$. The SR particles exhibit extensive porosity and cracking due to the removal of $\mathrm{Fe}$ from the precursor ilmenite. Figures $3 \mathrm{~b}-\mathrm{d}$ show that the addition of the much finer $\mathrm{Fe}_{2} \mathrm{O}_{3}$ powder caused the SR particles to be coated with $\mathrm{Fe}_{2} \mathrm{O}_{3}$, although not uniformly due to the angular morphologies of the powder.
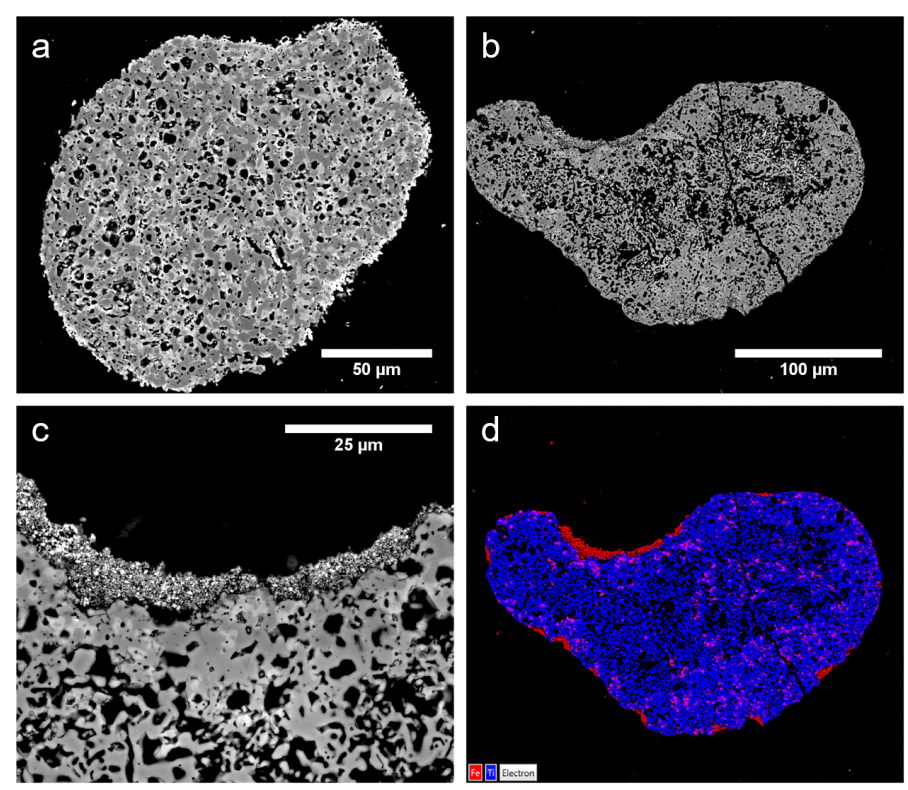

Figure 3: Backscattered electron micrographs: a) Standard SR particle, b) SR particle with $\mathrm{Fe}_{2} \mathrm{O}_{3}$, c) $\mathrm{Fe}_{2} \mathrm{O}_{3}$ on surface, d) X-EDS data of the particle in b) with $\mathrm{Ti}$ in blue and $\mathrm{Fe}$ in red

Electrolytically reduced synthetic rutile alloy powders

Elemental analysis of the resulting powders is listed in Table 1, with the alloys numbered by increasing Fe content. The $\mathrm{O}$ values were higher than is desirable for most commercial alloys; however, these are initial proof of principle experiments and optimisation of the electrolytic reduction is expected to improve this. The $\mathrm{C}$ values were suitably low meaning that carbide formation was avoided. 
Table 1: $\mathrm{O}, \mathrm{C}$ and $\mathrm{Fe}$ contents of the reduced alloy powders

\begin{tabular}{||c||c||c|c||}
\hline Alloy & O / ppm & C / ppm & Fe / wt. \% \\
\hline \hline 1 & 4825 & 227 & 3.7 \\
\hline \hline 2 & 4413 & 230 & 4.7 \\
\hline \hline 3 & 3750 & 269 & 5.3 \\
\hline \hline 4 & 4917 & 262 & 5.5 \\
\hline \hline
\end{tabular}

It was expected that each of the alloys would have distinct Fe contents due to the difference in the oxide compositions. This was not the case, however, and data showed that the Fe content range was narrower than predicted. This suggests that the Fe was able to migrate through the electrolyte between the separate sections during the Metalysis Process reduction. It is clear that the standard SR powder became contaminated with $\mathrm{Fe}_{2} \mathrm{O}_{3}$, as previous work showed that the alloy contained around 3.4 wt.\% total $\beta$ stabilising elements, compared with the 3.7 wt.\% of solely Fe in the SR derived Alloy 1 [9]. There is also significant Fe loss into the salt during the electrolytic reduction process, as the $5 \%$ composition should produce an alloy containing around $10 \mathrm{wt} \% \mathrm{Fe}$ if it is all retained. This could be partly prevented by altering the mixing process to improve adhesion of $\mathrm{Fe}_{2} \mathrm{O}_{3}$ to the SR.

Backscattered electron imaging of the alloy powders shows that there is a large variation in the microstructures present within the particles. This is expected as there is variation between the SR particles prior to reduction and imperfect mixing with $\mathrm{Fe}_{2} \mathrm{O}_{3}$ may lead to Fe rich regions and therefore an increased $\beta$ content. Figure 4 shows the microstructures of some of the SR derived particles which are typical in each of the four alloys studied. The particles still exhibit porosity, due to the nature of the SR feedstock and removal of oxygen, but less than that of the feedstock as some sintering occurs during the Metalysis Process reduction. 

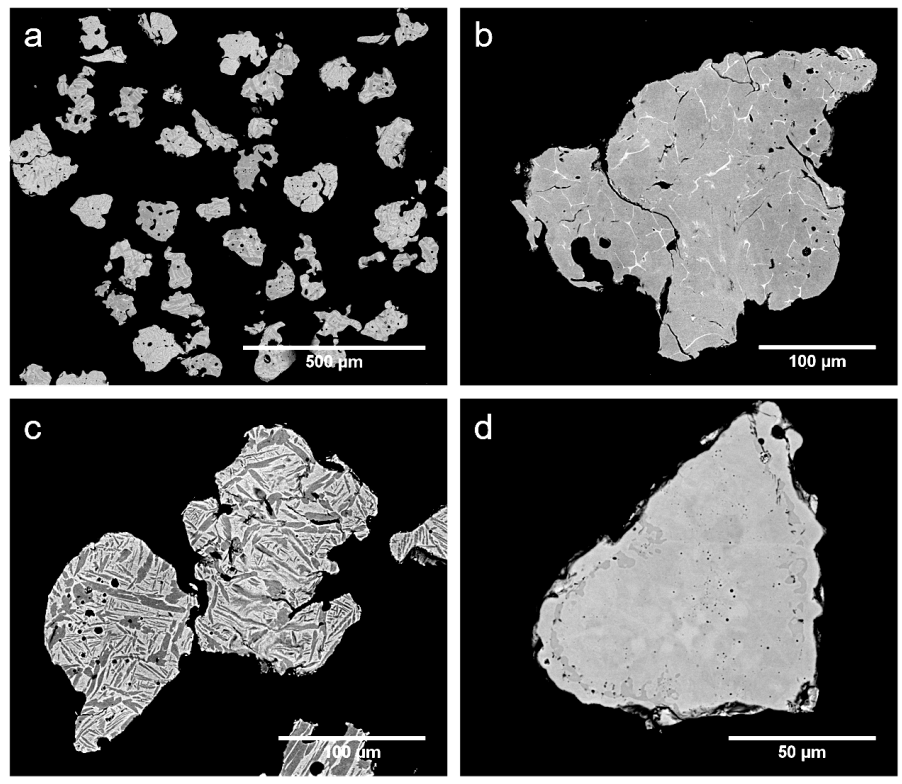

Figure 4: Backscattered electron micrographs of reduced SR alloy powders: a) range of particles, b) $\alpha$ rich particle, c) $\alpha+\beta$ particles, d) $\beta$ rich particle

\section{Consolidation of synthetic rutile derived alloys by field assisted sintering}

Figure 5 shows that consolidation of two of the alloys formed a homogeneous $\alpha+\beta$ microstructure, with no TiC or TiFe phases observed. Consolidation of the remaining alloys will allow for more microstructural comparison and it is expected that the higher Fe content alloys will have a greater $\beta$ content. It is important to note however that the higher than desired $O$ levels will have led to increased $\alpha$ phase volume fraction. The microstructure formed is also dependent on the cooling rate after the FAST dwell, but this was not controlled during the sintering and could be altered in the future. Some porosity was present, but this was expected as it is known that achieving full density with the initially porous powders is more challenging than when using non-porous commercial powders. Again, this could potentially be improved by modifying the FAST parameters. 

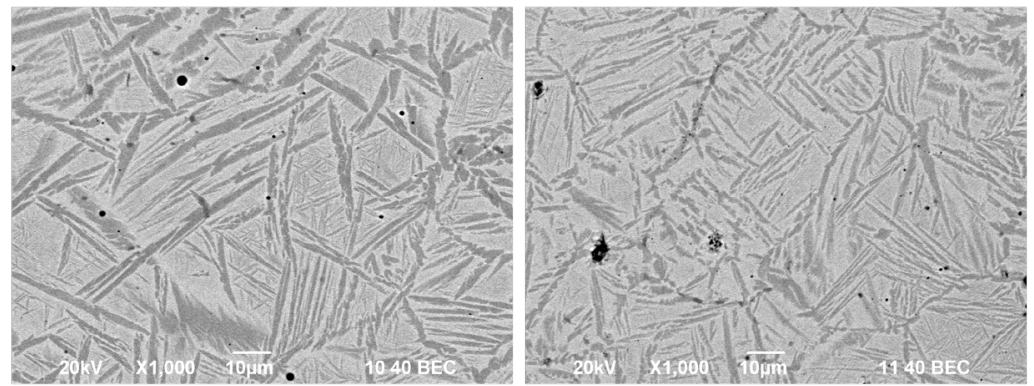

Figure 5: Backscattered electron micrographs of FAST consolidated alloy 1 (left) and 2 (right)

\section{$\underline{\text { Conclusion }}$}

Synthetic rutile was successfully co-reduced with $\mathrm{Fe}_{2} \mathrm{O}_{3}$ using the Metalysis Process to produce titanium alloys with $\mathrm{Fe}$ contents up to 5.5 wt.\%. Two of the powders were then consolidated using FAST to generate solid samples with homogeneous microstructures, however further work is required to fully compare all four of the alloys and thus the effect of Fe content. This includes larger scale FAST consolidation which would allow the production of samples for mechanical testing. Mechanical properties can then be compared with commercially available alloys and the potential range of applications assessed. Improved mixing of the oxides and optimisation of the reduction process can also be done to have greater control over the Fe content in the resulting alloys and reduce oxygen levels. For example, a heat treatment step, prior to the electrolytic reduction, will allow the Fe to diffuse into the SR rather than remaining on the surface. Alloys with higher Fe contents could then be produced to assess at what concentration TiFe formation begins to occur using this solid-state processing route. There is also potential to control the extent of Fe removal during the SR production from ilmenite, as this naturally occurring Fe will be within the particles and no additional oxide would be required to create the alloys.

\section{Acknowledgements}

The authors wish to thank EPSRC grant EP/L016273 Centre for Doctoral Training in Advanced Metallic Systems for supporting this research. We also acknowledge the technical staff at Metalysis, along with Ben Thomas and Nick Weston for help during field assisted sintering.

\section{References}

[1] G. Z. Chen, D. J. Fray, and T. W. Farthing, 'Direct electrochemical reduction of titanium dioxide to titanium in molten calcium chloride', Nature, vol. 407, no. 6802, pp. 361-364, Sep. 2000. 
[2] I. Mellor et al., 'Titanium powder production via the Metalysis process', in Titanium Powder Metallurgy, M. Qian and F. H. (Sam) Froes, Eds. Boston: Butterworth-Heinemann, 2015, pp. 51-67.

[3] R. Barnett, K. T. Kilby, and D. J. Fray, 'Reduction of Tantalum Pentoxide Using Graphite and Tin-Oxide-Based Anodes via the FFCCambridge Process', Metall. Mater. Trans. B, vol. 40, no. 2, pp. 150-157, Apr. 2009.

[4] E. Gordo, G. Z. Chen, and D. J. Fray, 'Toward optimisation of electrolytic reduction of solid chromium oxide to chromium powder in molten chloride salts', Electrochimica Acta, vol. 49, no. 13, pp. 2195-2208, May 2004.

[5] B. Claux, J. Serp, and J. Fouletier, 'Electrochemical reduction of cerium oxide into metal', Electrochimica Acta, vol. 56, no. 7, pp. 2771-2780, Feb. 2011.

[6] A. M. Martinez, K. S. Osen, H. Gudbrandsen, C. Sommerseth, Z. Wang, and O. Darell, 'Direct Method for Producing Scandium Metal and Scandium-Aluminium Intermetallic Compounds from the Oxides', Light Met. 2018, pp. 1559-1564, 2018.

[7] J. Sure, D. S. M. Vishnu, and C. Schwandt, 'Direct electrochemical synthesis of high-entropy alloys from metal oxides', Appl. Mater. Today, vol. 9, pp. 111-121, Dec. 2017.

[8] V. Hugo, 'Iluka’s Synthetic Rutile Production’, Iluka Resources Limited, Feb. 2012.

[9] L. L. Benson, I. Mellor, and M. Jackson, 'Direct reduction of synthetic rutile using the FFC process to produce low-cost novel titanium alloys', J. Mater. Sci., vol. 51, no. 9, pp. 4250-4261, May 2016.

[10] N. S. Weston and M. Jackson, 'FAST-forge - A new cost-effective hybrid processing route for consolidating titanium powder into near net shape forged components', J. Mater. Process. Technol., vol. 243, pp. 335-346, 2017.

[11] L. L. Benson, L. A. Benson Marshall, N. S. Weston, I. Mellor, and M. Jackson, 'On a Testing Methodology for the Mechanical Property Assessment of a New Low-Cost Titanium Alloy Derived from Synthetic Rutile', Metall. Mater. Trans. A, vol. 48, no. 11, pp. 5228-5232, Nov. 2017.

[12] A. Mitchell, 'Melting, casting and forging problems in titanium alloys', Mater. Sci. Eng. A, p. 6, 1998.

[13] W. Wei, Y. Liu, K. Zhou, and B. Huang, 'Effect of Fe addition on sintering behaviour of titanium powder', Powder Metall., vol. 46, no. 3, pp. 246-250, Oct. 2003.

[14] J.-O. Andersson, T. Helander, L. Höglund, P. Shi, and B. Sundman, 'Thermo-Calc \& DICTRA, computational tools for materials science', Calphad, vol. 26, no. 2, pp. 273-312, Jun. 2002.

[15] R. I. Jaffee, 'The Physical Metallurgy of Titanium Alloys', Prog. Met. Phys., vol. 7, pp. 65-163, 1958. 
[16] P. G. Esteban, L. Bolzoni, E. M. Ruiz-Navas, and E. Gordo, 'PM processing and characterisation of Ti-7Fe low cost titanium alloys', Powder Metall., vol. 54, no. 3, pp. 242-252, Jul. 2011.

[17] L. Bolzoni, E. M. Ruiz-Navas, and E. Gordo, 'Quantifying the properties of low-cost powder metallurgy titanium alloys', Mater. Sci. Eng. A, vol. 687, pp. 47-53, Feb. 2017.

[18] B.-Y. Chen, K.-S. Hwang, and K.-L. Ng, 'Effect of cooling process on the alpha phase formation and mechanical properties of sintered Ti-Fe alloys', Mater. Sci. Eng. A, vol. 528, no. 13-14, pp. 4556-4563, May 2011.

[19] N. S. Weston, F. Derguti, A. Tudball, and M. Jackson, 'Spark plasma sintering of commercial and development titanium alloy powders', J. Mater. Sci., vol. 50, no. 14, pp. 4860-4878, Jul. 2015. 